\title{
Dysregulation of core components of SCF complex in poly-glutamine disorders
}

\author{
S Bhutani, ${ }^{*, 1}$ A Das ${ }^{1}$, M Maheshwari ${ }^{1}$, SC Lakhotia ${ }^{2}$ and NR Jana ${ }^{1}$
}

Poly-glutamine (polyQ) diseases are neurodegenerative disorders characterised by expanded CAG repeats in the causative genes whose proteins form inclusion bodies. Various E3 ubiquitin ligases are implicated in neurodegenerative disorders. We report that dysfunction of the SCF (Skp1-Cul1-F-box protein) complex, one of the most well-characterised ubiquitin ligases, is associated with pathology in polyQ diseases like Huntington's disease (HD) and Machado-Joseph disease (MJD). We found that Cullin1 (Cul1) and Skp1, core components of the SCF complex, are reduced in HD mice brain. A reduction in Cul1 levels was also observed in cellular HD model and fly models of both HD and MJD. We show that Cul1 is able to genetically modify mutant huntingtin aggregates because its silencing results in increased aggregate load in cultured cells. Moreover, we demonstrate that silencing dCul1 and dSkp1 in Drosophila results in increased aggregate load and enhanced polyQ-induced toxicity. Our results imply that reduced levels of SCF complex might contribute to PolyQ disease pathology.

Cell Death and Disease (2012) 3, e428; doi:10.1038/cddis.2012.166; published online 22 November 2012

Subject Category: Neuroscience

Huntington's disease (HD) is an age-related neurodegenerative disorder, which manifests as motor, psychiatric and cognitive impairment. ${ }^{1}$ It belongs to a group of diseases called poly-glutamine (polyQ) diseases, all of which have expanded trinucleotide repeats (encoding glutamine) in their causative genes. HD is autosomal dominant and manifests when the number of CAG repeats in exon1 of Huntingtin gene exceeds $37 .^{2}$ Spinocerebellar Ataxia 3/Machado-Joseph disease (SCA3/MJD) also belongs to this category of neurodegenerative diseases and is caused by an expansion of CAG repeats in the Ataxin3 gene. ${ }^{3}$ Almost all neurodegenerative diseases, including poly $Q$ diseases, are characterised by the presence of protein aggregates, which are believed to be toxic and cause neuronal death. ${ }^{4}$

A variety of model systems are used to study the molecular basis of HD pathology in the hope of elucidating putative therapeutic targets. ${ }^{5}$ Non-mammalian systems like Drosophila and cellular models are useful to screen potential genetic modifiers of mutant huntingtin aggregates. For instance, overexpression of human Huntingtin gene exon1 with expanded CAG repeats in Drosophila results in the formation of protein aggregates and subsequent neurodegeneration. ${ }^{6,7}$ When expressed in the photoreceptor neurons of flies, age-related retinal degeneration is visible in the eye. In the cellular model, expression of mutant Huntingtin gene results in the formation of peri-nuclear aggregates. ${ }^{8}$ The $\mathrm{R} 6 / 2$ transgenic mouse model for $\mathrm{HD}$ is a well-established murine model that expresses mutant human Huntingtin exon1 having 150 CAG repeats. ${ }^{9}$ These mice show severe age-related neurodegeneration as assayed by biochemical and behavioural parameters.
The ubiquitin proteosome system (UPS) is one of the qualitycontrol systems used to maintain cellular integrity. Protein aggregates of various neurodegenerative diseases have been shown to be positive for ubiquitin, and the functioning of the UPS is believed to be compromised in these diseases. ${ }^{10,11}$ The UPS involves the functioning of three enzymes - E1, E2 and E3, in a cascading manner, which tag misfolded proteins with ubiquitin resulting in their subsequent degradation through the proteosome. Of these, the E3 ligases are responsible for substrate specificity. In addition, E3 ligases have been implicated in the pathogenesis of various neurodegenerative diseases, including polyQ diseases. ${ }^{12-17}$ The SCF complex (Skp1-Cul1-F-box protein) is one of the most well-characterised E3 ligases. In this complex, Cullin1 (Cul1) acts as a scaffold protein whose $\mathrm{N}$-terminal region binds Skp1, and C-terminal region binds a RING-finger protein called Rbx1. Skp1, in turn, can bind a variety of F-box proteins, which provide substrate specificity to the complex. ${ }^{18}$ For example, it is known that when the SCF complex binds F-box protein $\beta$-TrCP1, it degrades $\beta$ catenin. ${ }^{19}$ In addition, it has recently been shown that Nedd8, an $8-\mathrm{kDa}$ protein, binds Cul1, and this neddylation of Cul1 is important for its activity. ${ }^{20}$ The role of the SCF complex as a cell-cycle regulator in actively dividing cells is already well established. ${ }^{21}$ However, Cul1 and Skp1 are also present in postmitotic neuronal cells and their function is yet to be elucidated. $^{22,23}$ It has been demonstrated that knockdown of Cul1 in primary mouse midbrain, as well as cortical culture, renders neurons susceptible to death, suggesting that Cul1 may have a role in maintaining the integrity of post-mitotic neurons. ${ }^{24}$ This implies that their dysfunction could result in neurodegeneration.

\footnotetext{
${ }^{1}$ Department of Cellular and Molecular Neurosciences, National Brain Research Centre, Gurgaon 122 050, India and ${ }^{2}$ Department of Zoology, Cytogenetics Laboratory, Banaras Hindu University, Varanasi 221005, India

${ }^{*}$ Corresponding author: S Bhutani, Department of Cellular and Molecular Neurosciences, National Brain Research Centre, NH-8, Manesar, Gurgaon 122 050, India. Tel: +91 9818948013; Fax: +91 1242338910; E-mail: supriya@nbrc.ac.in

Keywords: Cullin1; E3 ligase; Huntington's disease; neurodegeneration; SCF

Abbreviations: SCF, Skp1-Cul1-F-box protein; HD, Huntington's disease; MJD, Machado-Joseph disease; SCA3, Spinocerebellar Ataxia 3; polyQ, poly-glutamine; UPS, ubiquitin proteosome system; tNhtt, truncated N-terminal huntingtin

Received 10.5.12; revised 27.9.12; accepted 5.10.12; Edited by D Bano
} 
Mandel and colleagues ${ }^{25-28}$ have shown that silencing Skp1 in models of Parkinson's disease leads to impaired functioning and survival of dopaminergic neurons and results in the formation of aggregates similar to Lewy bodies, which are hallmarks of the disease. However, little information is available about the status of SCF complex in polyQ disorders like HD, although core components as well as interacting partners of the SCF complex like cul1, Skp1 and nedd8 are among the many genes identified as modifiers of mutant huntingtin aggregates in Drosophila, in two independently conducted RNAi screens. ${ }^{29,30}$

In this study, we have investigated the status of Cul1 and Skp1 of the SCF complex in cellular, fly and murine HD models. We have also genetically manipulated Cul1 and Skp1 in Drosophila and shown a consequent modulation of the disease phenotype. Our findings in HD have been confirmed in fly models for both MJD/SCA3 and expanded polyQ repeats. Together, our results suggest that lowered activity of the SCF complex aggravates polyQ disease pathogenesis.

\section{Results}

Levels of Cul1 and Skp1 proteins are reduced in R6/2 transgenic mouse model of HD. Upon investigating the status of core components of the SCF complex in the brain of wild-type mice, we found that endogenous Cul1 and Skp1 protein were detectable in immunoblot of lysates made from cortex and cerebellum of both 6-8- and 12-14-weeks-old mice. When levels of Cul1 protein were compared between HD mice and their age-matched wild-type controls, a significant $30 \%$ reduction was observed in HD mice at both ages (Figures 1a and b; blot only shown for 6-8-weeks-old mice). In HD, numerous proteins are found to be reduced in the cytosol because they get recruited to insoluble aggregates. When brain sections are stained for these proteins, aggregates are visible as dense structures in the nucleus (Supplementary Figure 1). In order to check if the reduction in soluble Cul1 was also due to its recruitment to aggregates, immunohistochemistry was done on mice brain sections using Cul1 antibody (Figure 1c). Although a reduced expression of Cul1 protein was evident in cortical and cerebellar sections, Cul1 was not found to co-localise with mutant huntingtin aggregates.

Further, because Skp1 is known to be an integral part of the SCF complex and to interact with Cul1, we assayed its levels in wild-type and HD mice. Similar to Cul1, a significant 30\% reduction in Skp1 levels was observed in cortex and cerebellum of HD mice aged 6-8 weeks in immunoblot (Figures $2 \mathrm{a}$ and $\mathrm{b}$ ) and immunohistochemistry on brain sections (Figure 2c). Reduced levels of members of the SCF complex should result in accumulation of its substrates. In agreement, $\beta$-catenin was found to accumulate in cortex of 6-8-week-old HD mice (Supplementary Figure 2).

Cul1 levels are reduced in cellular HD model and its overexpression has a dominant-negative effect on mutant huntingtin aggregation. We used a well-established cellular model of $\mathrm{HD}$, where truncated $\mathrm{N}$-terminal huntingtin (tNhtt) with $150 \mathrm{Q}$ repeats fused to a
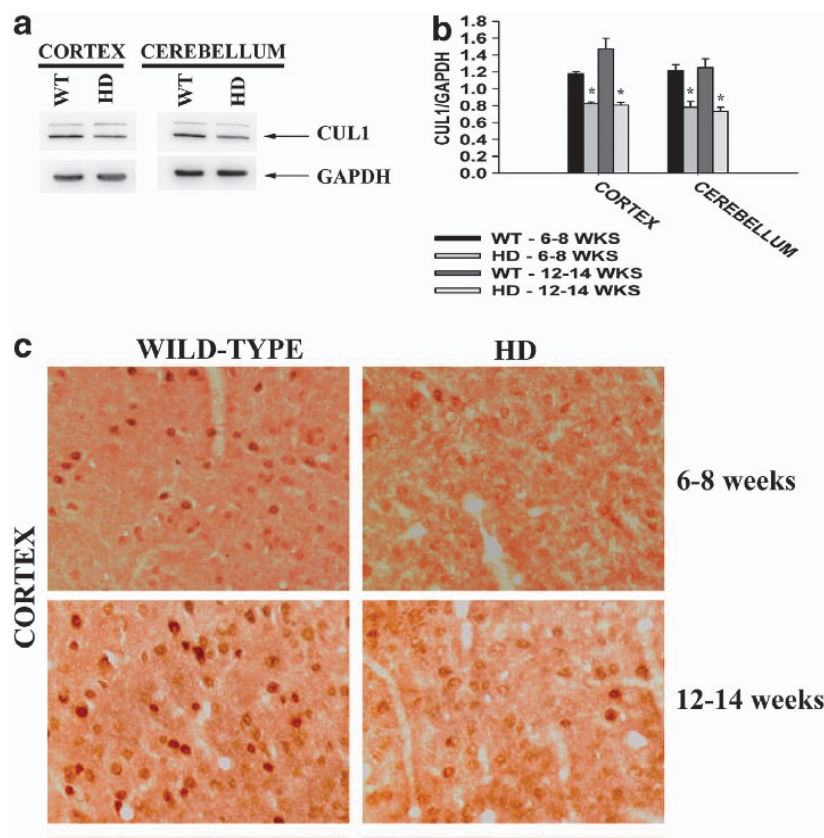

6-8 weeks
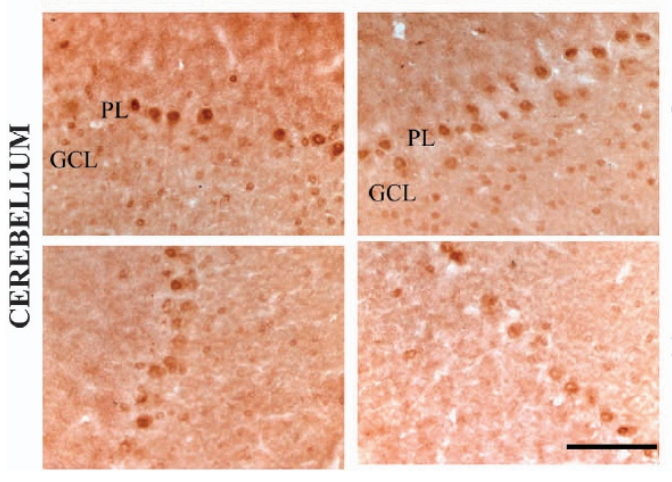

6-8 weeks

Figure 1 Endogenous Cul1 in R6/2 transgenic HD mice brain. (a) Immunoblot of Cul1 in lysates of cortex and cerebellum of wild-type and HD mice aged 6-8 weeks. GAPDH used as loading control. (b) Quantification of Cul1 in cortex and cerebellum of HD mice brains. Quantification was done using NIH Image analysis software. Values are mean \pm S.E.M of three different mice in each group. " $P<0.05$ with respect to control (one-way ANOVA). (c) Immunohistochemistry on brain sections of 6-8- and 12-14-week-old mice using Cul1 antibody. There is a reduction of Cul1 protein levels in HD mice brain but no co-localisation to huntingtin aggregates. GCL, granular cell layer; PL, Purkinje layer. Images were taken using $\times 40$ objective (Scale bar: $20 \mu \mathrm{m}$ )

GFP was inducibly expressed. ${ }^{8}$ The cell line was named HD-150Q. Endogenous levels of Cul1 were assayed in HD-150Q cells in which mutant huntingtin was induced for $12 \mathrm{~h}$ using $1 \mu \mathrm{M}$ ponasterone $\mathrm{A}$ and compared with uninduced controls. A highly significant reduction in Cul1 levels (60\%) was observed upon induction of mutant huntingtin (Figures 3 a and b). This reduction was also visible through immunostaining because cells with aggregates revealed reduced levels of Cul1 compared with those without them (Figure 3c). As seen in mice brain sections, endogenous Cul1 was not found to be localised with huntingtin aggregates.

It has been reported that overexpressed truncated as well as full-length Cul1 act in a dominant-negative manner and reduce activity of the endogenous protein in Drosophila C. elegans, as well as in mammalian cell culture. ${ }^{31,32}$ 
a
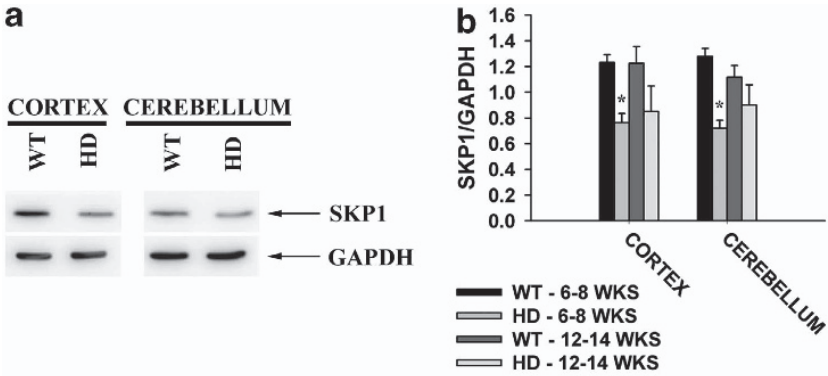

C WILD-TYPE
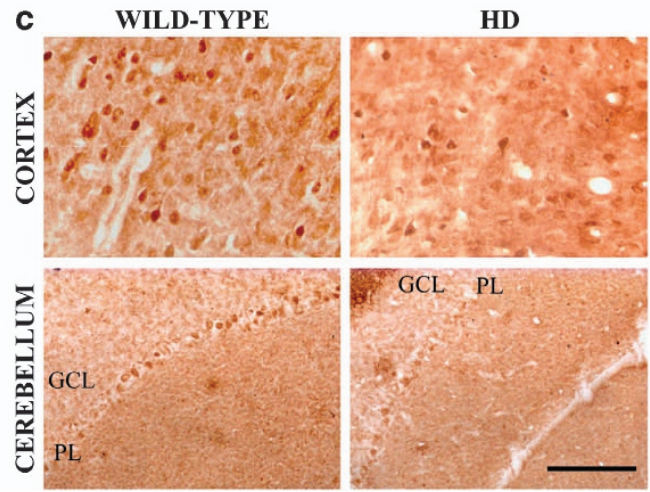

Figure 2 Endogenous Skp1 in HD mice brain. (a) Immunoblot of Skp1 in lysates of cortex and cerebellum of wild-type and HD mice aged 6-8 weeks. GAPDH used as loading control. (b) Quantification of Skp1 in the cortex and cerebellum of wildtype and HD mice brains. Values are mean \pm S.E.M; $n=3$. ${ }^{*} P<0.05$ with respect to wild-type mice (one-way ANOVA). (c) Immunohistochemical staining of Skp1 in 6-8-week-old wild-type and HD mice brain sections. GCL, granular cell layer; PL, Purkinje layer. Images were taken using $\times 40$ objective (Scale bar: $20 \mu \mathrm{m}$ )

Therefore, we transfected HD-150Q cells with full-length human Cul1 (Figure $3 \mathrm{~d}$ ) to check if aggregate load is affected following increase in the level of Cul1. For this, the number of aggregates in transfected cells was manually counted and compared with that in cells transfected with empty vector. The percentage of transfected cells containing aggregates was found to be significantly higher than in controls (Figure 3e). The fact that overexpressing hCul 1 increases aggregate load in cell culture implies, in agreement with earlier studies, ${ }^{25,26}$ that it is acting in a dominant-negative manner and Cul1 is indeed able to genetically modify mutant huntingtin aggregates. Therefore, we investigated this possibility by using the Drosophila HD model.

Modulating dCul1 and dSkp1 levels in Drosophila. To check the effect of overexpressing full-length human Cul1 in flies, we generated transformant flies by cloning hCul1 from pcDNA3 into pUASTattB and performing site-directed transformation (See Materials and Methods). In parallel, RNAi lines were obtained from VDRC, Vienna, to silence endogenous dCul1 and dSkp1 expression. GMR-GAL4-driven expression of these transgenes in an eye-specific manner did not cause any lethality (Table 1a), but in both dCul1 modifications, the eyes showed rough phenotype compared with the appropriate parental controls (Figure 4a). Surprisingly, silencing dSkp1 in the eyes using RNAi did not result in a roughened retina. The external morphology of the eye is visible in photographs as well as nail polish imprints. Nail polish imprints of normal eyes depict organised ommatidial arrangement, which is disrupted in flies downregulating dCul1 or overexpressing hCul1 but not in those downregulating dSkp1 (Figure 4a). Protein extracted from heads of the flies revealed the expected increase or decrease in Cul1 levels when compared with wild-type (OregonR ${ }^{+}$) (Figure 4b), and also the expected reduction in dSkp1 levels upon RNAi as compared with controls (Figure 4c).

dCul1 protein and RNA levels are reduced in a fly model of HD. Human Huntingtin gene exon1, containing 93 polyQ repeats (HTT-EXON1-PQ93), was expressed in the eyes of flies using the GMR-GAL4 driver. The flies were aged for 15 days to allow retinal degeneration to take place. When head lysates of these flies were probed for endogenous dCul1, a significant (about $30 \%$ ) reduction in levels of dCul1 protein was observed in HD flies as compared with age-matched GMR-GAL4 controls (Figure 5a). The dCul1 RNA is also significantly reduced in HD flies (Figure $5 \mathrm{~b}$ ), indicating that expression of expanded polyQ protein affects dCul1 gene transcription.

Silencing dCul1 accelerates disease pathogenesis in HD flies. In order to assay whether dCul1 can modify HD pathogenesis in flies, dCul1 RNAi was coexpressed with HTT-EXON1-PQ93 under the control of GMR-GAL4 driver. Surprisingly, this cross resulted in lethality of flies as only $9 \%$ of the progeny that eclosed contained all three transgenes as opposed to the expected $25 \%$ (Table $1 \mathrm{~b}$ ). Also, the eyes of all surviving adults had a roughened retina with enhanced degeneration, resulting in a bulbous structure on the eye (arrow in Figure 5c). The ommatidial arrays, as revealed by the nail polish imprints, ${ }^{33}$ were much more damaged than in GMR-GAL4/ UASHTT-EXON1-PQ93 control flies.

dCul1 protein and RNA levels are reduced in a fly model of MJD and silencing it enhances retinal degeneration. MJD is another neurodegenerative disease caused by an expanded polyQ repeat in Ataxin3. GMR-GAL4-driven overexpression of human Ataxin3 with 78 polyQ repeats results in retinal degeneration and serves as a model for another poly $Q$ disease in flies (MJD flies). ${ }^{34}$ Similar to HD, both dCul1 protein and RNA levels were reduced in MJD flies compared with controls (Figures $6 \mathrm{a}$ and $\mathrm{b}$ ). In addition, when dCul1 RNAi was coexpressed with UASMJD-PQ78(s), it resulted in lethality of the flies (Table 1b), and the few GMR-GAL4/ UASMJD-PQ78(s);UASdCUL1-RNAi adults that emerged showed enhanced degeneration compared with control flies and appearance of dense black spots in the eye (Figure 6c).

Silencing dSkp1 in HD and MJD flies enhances retinal degeneration. In order to confirm that the enhancement of $\mathrm{HD}$ and MJD pathogenesis following manipulation in dCul1 levels was because of its role in the SCF complex, dSkp1, another core component of SCF was silenced using RNAi. dSkp1 RNAi in HD fly model resulted in lethality (Table 1b), and surviving adults had enhanced retinal degeneration (Figure 7a). In MJD flies, no drastic lethality was observed, instead all GMR-GAL4/UASMJD-PQ78(s);UASdSKP1-RNAi 

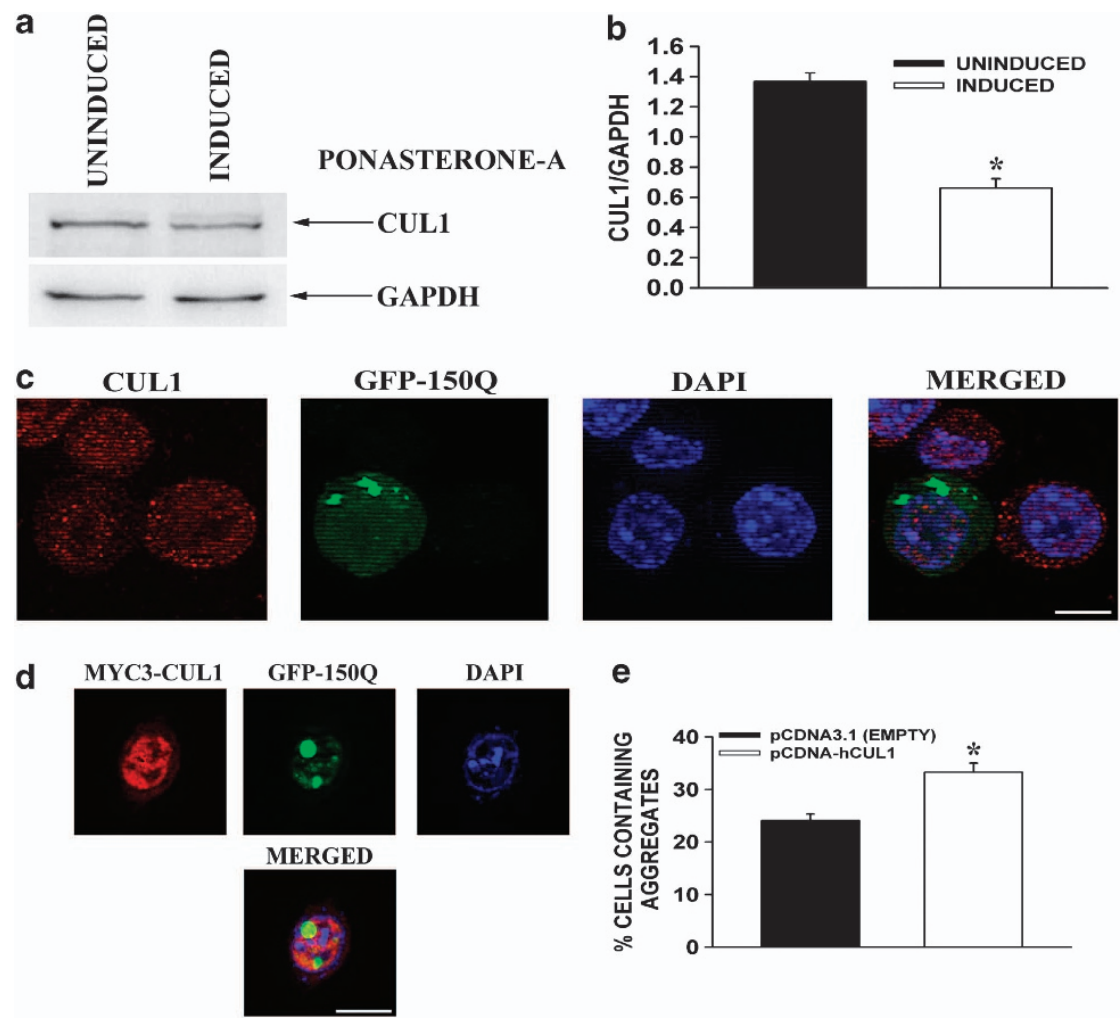

e

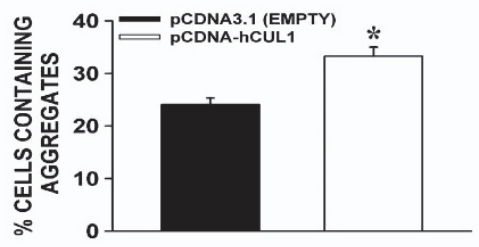

Figure 3 Cul1 in a cellular model of HD. (a) Immunoblot analysis of Cul1 in HD-150Q cells in which mutant huntingtin was induced for $12 \mathrm{~h}$ with ponasterone $\mathrm{A}(1 \mu \mathrm{M})$. GAPDH used as loading control. (b) Quantification of Cul1 shows a significant reduction ( $P<0.001 ; n=5)$ of endogenous protein levels upon induction of mutant huntingtin as compared with uninduced control cells. (c) Fluorescence staining of Cul1 in HD-150Q cells showing a decrease in its level. Scale bar: $10 \mu \mathrm{m}$. (d) HD-150Q cells were transfected with myc-tagged hCul1 for $24 \mathrm{~h}$. Mutant huntingtin was induced for $12 \mathrm{~h}$ and then subjected to immunofluorescence staining of myc to detect overexpressed $\mathrm{hCul} 1$. Scale bar: $10 \mu \mathrm{m}$. (e) Counting of aggregates shows that the percentage of hCul1-transfected cells containing aggregates is significantly more ${ }^{*} P=0.015 ; n=3$, Student's $t$ test) than control cells

Table 1 Overexpressing hCul1 and downregulating dCul1 or dSkp1 in Drosophila results in lethality in the presence of polyQ

\begin{tabular}{|c|c|c|c|c|}
\hline Desired Genotype & $\begin{array}{l}N(\text { Total adults } \\
\text { eclosed) }\end{array}$ & $\begin{array}{c}n \text { (Desired adults } \\
\text { eclosed) }\end{array}$ & $\begin{array}{l}\text { \% Expected } \\
\text { genotype }\end{array}$ & $\begin{array}{c}\% \text { Observed } \\
\text { genotype }\end{array}$ \\
\hline \multicolumn{5}{|l|}{$A$} \\
\hline GMR-GAL4;UASdCUL1-RNAi (42445) & 105 & 105 & 100 & 100 \\
\hline GMR-GAL4;UASdCUL1-RNAi (33406) & 72 & 72 & 100 & 100 \\
\hline GMR-GAL4;UASdCUL1-RNAi (108558) & 85 & 85 & 100 & 100 \\
\hline GMR-GAL4;UAShCUL1 & 130 & 72 & 50 & 55 \\
\hline GMR-GAL4;UASdSKP1-RNAi (46605) & 128 & 128 & 100 & 100 \\
\hline GMR-GAL4;UASdSKP1-RNAi (46607) & 110 & 110 & 100 & 100 \\
\hline \multicolumn{5}{|l|}{$B$} \\
\hline GMR-GAL4/UASHTT-EX1-PQ93; UASdCUL1-RNAi & 174 & 17 & 25 & 9.7 \\
\hline GMR-GAL4/UASMJD-PQ78(s); UASdCUL1-RNAi & 215 & 5 & 25 & 2.3 \\
\hline GMR-GAL4/UASHTT-EX1-PQ93; UASdSKP1-RNAi & 152 & 3 & 25 & 1.9 \\
\hline GMR-GAL4/UASMJD-PQ78(s); UASdSKP1-RNAi & 200 & 38 & 25 & 19 \\
\hline \multicolumn{5}{|l|}{ C } \\
\hline GMR-GAL4.UAS127Q;UASdCUL1-RNAi (42445) & 404 & 10 & 50 & 2 \\
\hline GMR-GAL4.UAS127Q; UASdCUL1-RNAi (33406) & 107 & 0 & 50 & 0 \\
\hline GMR-GAL4.UAS127Q;UASdCUL1-RNAi (108558) & 246 & 9 & 50 & 4 \\
\hline GMR-GAL4.UAS127Q;UAShCUL1 & 118 & 0 & 50 & 0 \\
\hline GMR-GAL4.UAS127Q;UASdSKP1-RNAi (46605) & 42 & 2 & 50 & 4.7 \\
\hline GMR-GAL4.UAS127Q; UASdSKP1-RNAi (46607) & 74 & 1 & 50 & 1.3 \\
\hline
\end{tabular}

flies that emerged had black spots on the eyes (Figure 7b). Thus, dSkp1 knockdown also enhances the toxicity of polyQ diseases, indicating the involvement of the SCF complex in pathogenesis of these diseases.
Silencing dCul1 and dSkp1 increases aggregate load and enhances polyQ toxicity. We wanted to check whether the detrimental effect of silencing dCul1 and dSkp1 is limited to HD and MJD or could possibly be extended to 
a

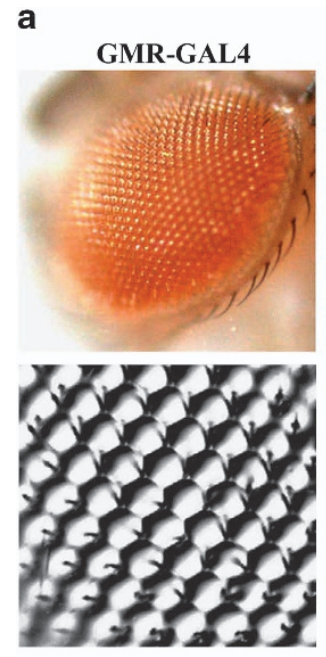

GMR-GAL4; UAShCUL1
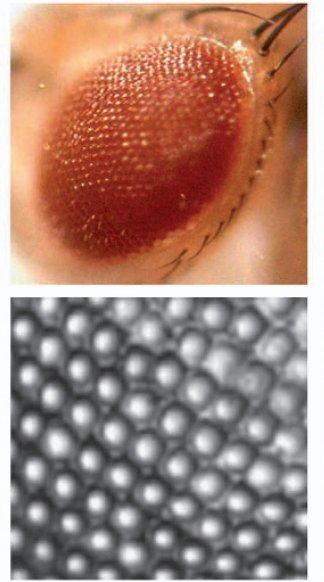

GMR-GAL4; UASdCUL1-RNAi
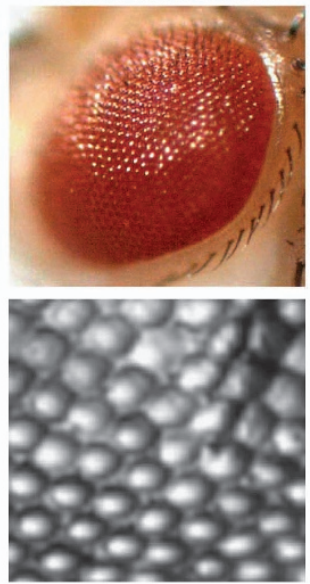

GMR-GAL4; UASdSKP1-RNAi
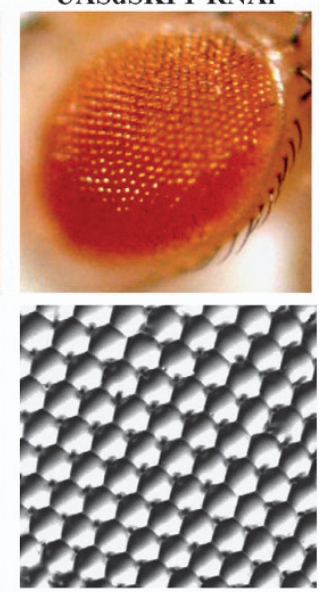

b

c

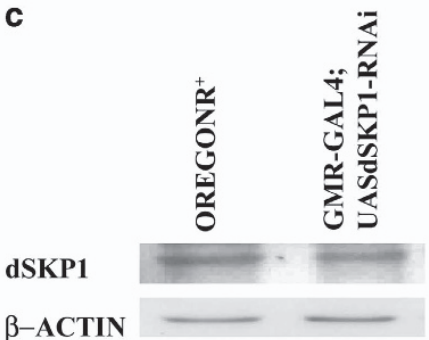

$\beta-$ ACTIN

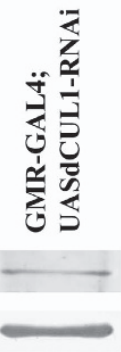

$\beta-\mathbf{A C T I N}$

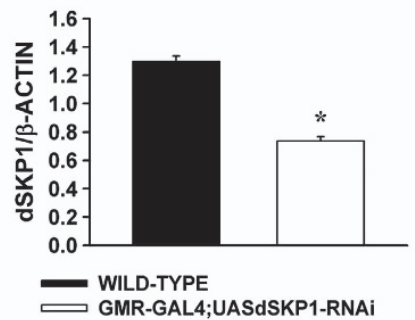

Figure 4 Modulating dCul1 and dSkp1 levels in Drosophila eyes. (a) Top panel - External morphology of the eye surface of Drosophila expressing either dCul1 RNAi or UAShCul1 in the eyes shows slightly roughened eyes compared with control. dSkp1 RNAi in the eyes does not result in a roughened retina. Bottom panel - The nail polish imprint of control shows parallel arrays of hexagonal ommatidia and this regular structure is lost when dCul1 is silenced or hCul1 is overexpressed but not when dSkp1 is knocked down. (b) Immunoblot analysis and quantification of fold change in dCul1 on head extracts of control flies (lane 1) compared with flies overexpressing (lane 2 , ${ }^{\star} P=0.0005 ; n=3$ ) and downregulating dCul1 (lane $3,{ }^{\star} P=0.001 ; n=3$ ). A one-way ANOVA was used followed by Holm-Sidak post-hoc test. $\beta$-actin used as loading control. (c) Immunoblot and quantification of fold change in dSkp1 on head extracts of control flies (lane 1) compared with flies downregulating dSkp1 (lane 2, ${ }^{*} P=0.0004$ using Student's $t$-test; $n=3$ ). $\beta$-actin used as loading control

polyQ diseases in general. For this purpose, active dCul1 was reduced by overexpressing dominant-negative hCul1 or by using dCul1 RNAi in flies expressing HA-tagged 127 glutamine repeats (127Q) under the control of GMR-GAL4 driver. Similarly, dSkp1 was reduced by RNAi in GMRGAL4.UAS127Q flies. These crosses resulted in a more extreme phenotype. Very few (0-4\% instead of the expected $50 \%$ ) of the progeny were found to contain all three transgenes (Table 1c). The robust eclosion of flies with the RNAi or overexpression transgene and balancer chromosome from the same cross-served as an internal control for growth conditions.
Examination of eyes of the surviving GMR-GAL4.UAS127Q;UASdCUL1-RNAiand GMR-GAL4.UAS127Q;UASdSKP1RNAi flies (no GMR-GAL4.UAS127Q;UAShCUL1 adults eclosed) revealed an exaggeration of eye damage when compared with GMR-GAL4.UAS127Q controls (Figure 8a). This damage was visible at the level of gross external eye morphology as well as ommatidial arrangement. We assayed the effect of silencing dCul1 and dSkp1 on polyQ aggregates by staining the late third instar larval eye discs with HA antibody. The aggregate load was significantly higher when active dCul1 levels were reduced either by dCul1 RNAi or hCul1 overexpression, or dSkp1 was reduced by RNAi 
a
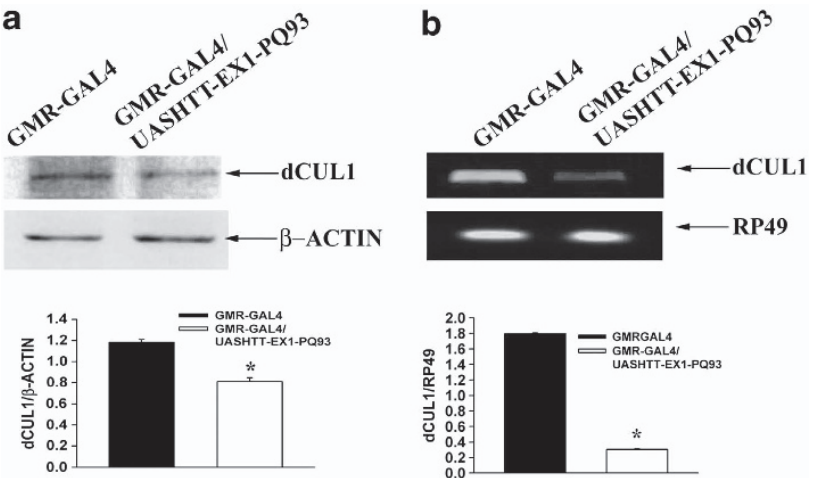

C GMR-GAL4/
UASHTT-EX1-PQ93
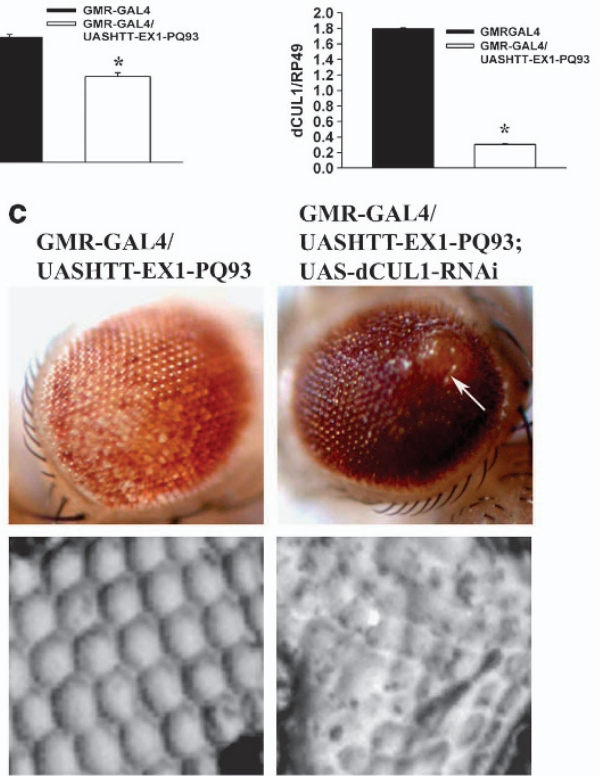

GMR-GAL4/

UASHTT-EX1-PQ93;

UAS-dCUL1-RNAI
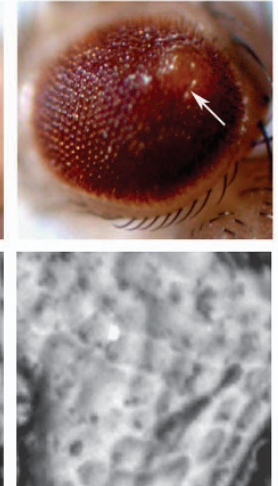

Figure 5 dCul1 in HD flies. (a) Immunoblot analysis of endogenous dCul1 in head lysates of 15 -day-old HD flies compared with age-matched controls. $\beta$-actin used as loading control. Quantification of dCul1 band intensities shows a significant reduction in its level ( $P=0.0013$ using Student's $t$-test; $n=5)$. (b) RT-PCR analysis of dCul1 transcript in head lysates of 15-day-old HD flies compared with age-matched controls. RP49 used as loading control. Quantification shows a significant reduction ( ${ }^{*} P<0.001$ using Student's $t$-test; $\left.n=5\right)$ of the dCul1 transcript. (c) External morphology (top panel) and nail polish imprints (bottom panel) of the eye surface of Drosophila with dCul1 RNAi in the presence of Httexon1 containing 93 polyQ repeats compared with control. RNAi of dCul1 enhances retinal degeneration (arrow) in HD flies as visible in photographs of the eyes, as well as in disruption of ommatidial arrays

(Figure 8b). These observations indicate a dependence of polyQ aggregate load and toxicity on the functioning of the SCF complex in Drosophila.

\section{Discussion}

Recently, dCul1, along with dCul3, has been implicated in development of the eye in Drosophila. ${ }^{35}$ Thus, it is not surprising that silencing dCul1 in the eye of flies results in a slightly roughened retina. However, intriguingly dSkp1-RNAi in the eye did not result in roughening of the eye. In their work, Ou et al. ${ }^{35}$ have addressed the role of dCul1 and dNedd8 in the developing eye imaginal disc through mutant and clonal analysis but have not reported any damage in the eyes of adults. In this work, we have not analysed any defects in developing eye imaginal discs, and thus our work does not exclude a role of dSkp1 in eye development. However, it also does not exclude the fact that dCul1 could be acting a

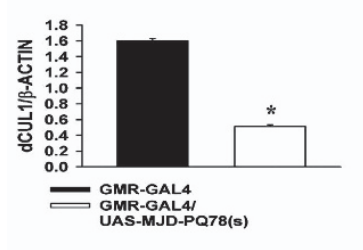

C GMR-GAL4/ UASMJD-PQ78(s)
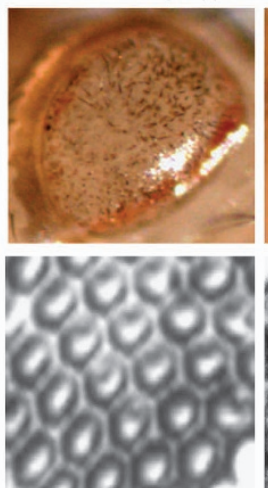

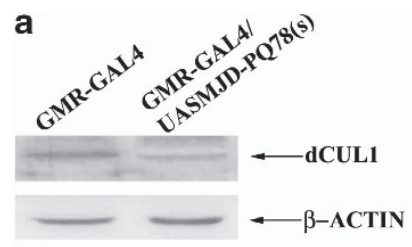

b
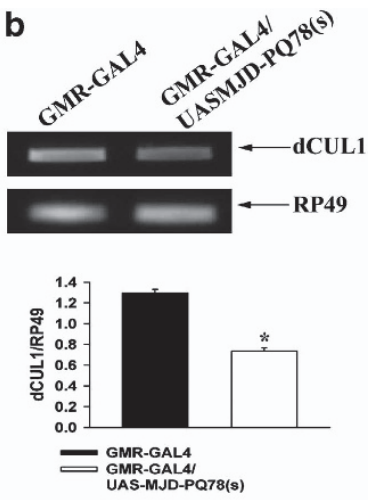

GMR-GAL4/

UASMJD-PQ78(s);
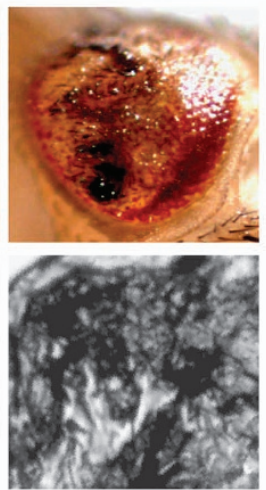

UAS-dCUL1-RNA

Figure 6 dCul1 in MJD flies. (a) Immunoblot analysis of endogenous dCul1 in head lysates of MJD flies compared with control. $\beta$-actin used as loading control. Quantification shows a significant reduction $\left({ }^{*} P<0.001\right.$ using Student's $t$-test; $n=3$ ) of dCul1 protein. (b) RT-PCR analysis of dCul1 transcript in head lysates of MJD flies compared with control. RP49 used as loading control. Quantification shows a significant reduction ( $P<0.001$ using Student's $t$-test; $n=5)$ of the dCul1 transcript. (c) External morphology (top panel) and nail polish imprints (bottom panel) of the eye surface of Drosophila with dCul1 RNAi in the presence of 78 polyQ repeats in Ataxin3 compared with control. RNAi of dCul1 enhances retinal degeneration in MJD flies, which is visible as dark black spots on the eyes in the photographs, as well as in disruption of ommatidial arrays

independent of dSkp1 in the process of the development of the eye in flies.

Silencing dCul1 by RNAi or overexpression of dominantnegative hCul1 and silencing dSkp1 by RNAi in the eyes does not affect eclosion of adult flies, indicating that these constructs are not lethal when expressed in the eyes by themselves. Thus, it was surprising to note that silencing dCul1 and dSkp1 in the eyes in polyQ disease models of HD, SCA3 and an artificial HA-tagged polyQ construct resulted in lethality of flies. This lethality indicates that polyQ toxicity is enhanced and this is confirmed by the fact that surviving adults show worsened retinal degeneration than controls. Enhancement of the GMR-GAL4-driven polyQ toxicity in certain other genotypes is also known to result in high pupal lethality. ${ }^{7}$ Larval eye imgainal discs show increased aggregate load upon reduction of Skp1 by RNAi and dCul1 by RNAi or overexpression of dominant-negative hCul1, which parallels the exaggerated retinal degeneration seen in surviving adult flies. An enhanced aggregate load upon expressing a 
a
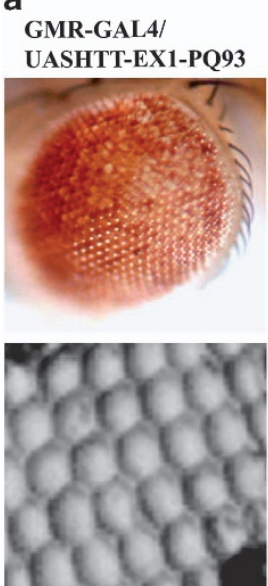

GMR-GAL4/

UASHTT-EX1-PQ93,

UASdSKP1-RNAi
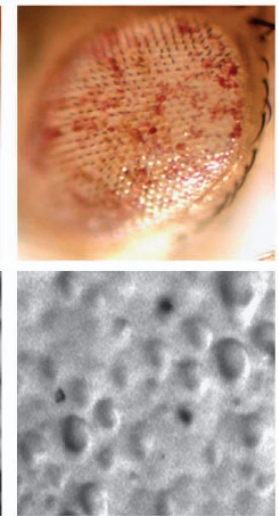

b

GMR-GAL4/ UASMJD-PQ78(s)
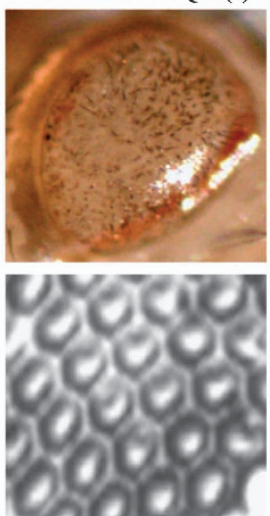

GMR-GAL4/

UASMJD-PQ78(s);

UASdSKP1-RNAi
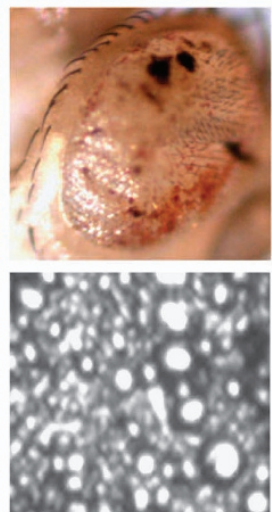

Figure 7 Effect of silencing dSkp1 in HD and MJD flies. (a) Silencing dSkp1 in HD flies results in enhanced degeneration in the form of large black spots, which is visible in external morphology (top panel) and nail polish imprints (bottom panel) of the eye. (b) Silencing dSkp1 in MJD flies enhances degeneration, and black spots are visible in the eyes. External morphology (top panel) and nail polish imprints (bottom panel) of the eye are shown

dominant-negative hCul1 construct is also observed in cell culture, indicating that this effect of SCF complex on mutant huntingtin aggregates is not unique to the fly model.

In this work, we have established that endogenous Cul1 protein is reduced in murine, cellular and fly models of $H D$. It is also reduced in a fly model of MJD. This reduction of protein levels is probably occurring at the transcriptional level because the abundance of dcul1 mRNA transcript was found to be greatly reduced in fly models of both diseases. Transcriptional dysregulation is believed to be a hallmark of polyQ diseases possibly because of the fact that various transcription factors are sequestered by the aggregates. ${ }^{36}$ The disruption of SCF function in HD and MJD, as reported in this work, is expected to result in an accumulation of its substrates. SCF complex potentially has a wide network of substrates because of the fact that it can bind with as many as 70 F-box proteins. ${ }^{18}$ Also, most of its known substrates, such as $\beta$-catenin, $\mathrm{P} 21, \mathrm{P} 27$ and cyclin $\mathrm{E}$, are involved in regulation of the cell cycle. ${ }^{37}$ It is believed that in neurodegenerative diseases, dysregulation of the cell cycle also contributes to apoptosis and neuronal death. ${ }^{38}$ As expected because of the reduced levels of components of the E3 ligase, SCF and subsequent accumulation of its target cell-cycle-related proteins, an increase in levels of $\beta$-catenin has been reported in HD patients, as well as in cellular, fly and murine models of HD, and other neurodegenerative diseases. ${ }^{39}$ Further, Godin and colleagues ${ }^{19}$ showed that the deleterious effect of mutant huntingtin aggregates can be obliterated in Drosophila by overexpressing Slimb, the Drosophila homologue of $\beta-T r C P$, which, in conjugation with the SCF complex, results in the degradation of $\beta$-catenin. Our work suggests that various other accumulated SCF substrates could also be contributing to toxicity and neuronal death and, therefore, they could also be potentially targeted for therapeutic purposes.

Some previous studies have suggested a correlation between SCF and neurodegenerative diseases, including polyQ diseases like MJD and dentatorubral-pallidoluysian atrophy. For example, Nedd8, a protein which is indispensible for the activity of Cul1, has been found to co-localise with inclusion bodies in brain sections from various neurodegenerative disease patients. ${ }^{40,41}$ In addition, it has been reported that silencing cul1, skr1 and $r b x 1$ (C. elegans homologues of components of SCF complex) enhances polyQ-induced paralysis in nematodes. ${ }^{42}$ In this work, we show that Cul1 and Skp1 modify neurodegeneration in HD, MJD and artificial polyQ expansion in fly models. In view of the reports in other polyQ disease models, and our data reported here, it is likely that both Cul1 and Skp1 are involved in other neurodegenerative diseases as well. Exploring the role of SCF complex in the fly and other models of these other diseases could thus provide some insight into common aspects of their pathogenesis.

\section{Materials and Methods}

HD transgenic mice. Ovarian-transplanted hemizygote females carrying htt exon1 with approximately 150 CAG repeats (strain name - B6CBA$\mathrm{Tg}(\mathrm{HDexon} 1) 62 \mathrm{Gpb} / 3 \mathrm{~J}$ ) were obtained from Jackson Laboratory (Bar Harbor, $\mathrm{ME}, \mathrm{USA}$ ) and were maintained by crossing with B6CBAF1/J males. All animal experiments were conducted in accordance with the approval of the Institutional Animal Ethics Committee of National Brain Research Centre, Manesar. Genotyping was carried out using a previously described method. ${ }^{9}$ Animals were killed using cervical dislocation, brains were carefully dissected and stored at $-80^{\circ} \mathrm{C}$.

Immunohistochemistry. Transgenic (R6/2) and wild-type mice of 6-8 and 12-14 weeks of age were anesthetised and perfused with 4\% PFA. The brains were then dissected and processed for cryosectioning to obtain sections with a thickness of $20 \mu \mathrm{m}$. The sections were then processed for immunohistochemistry using reagents from Vector Laboratories (Burlingame, CA, USA). Briefly, after retrieval of antigen by 45 -min incubation at $70^{\circ} \mathrm{C}$, sections were blocked and probed using rabbit anti-Cul1 (1:100, Abcam, Cambridge, MA, USA), rabbit antiSkp1 (1:100, Cell Signalling, Danvers, MA, USA) or rabbit anti-ubiquitin (1:500, Dako, Glostrup, Denmark). Biotinylated secondary antibodies were used at a dilution of $1: 250$, and signal was enhanced using ABC kit (Vector Laboratories). The staining was visualised using ImmPACT Novared Peroxidase Substrate (Vector Laborations). Images were taken in bright field using a Leica (Wetzlar, Germany) DM RXA2 microscope and $\times 40$ objective.

Cell culture. A stable HD-150Q cell line, containing a ecdysone-inducible pIND-tNhtt-150Q-GFP construct, has already been described. ${ }^{8}$ Cell-culture media and fetal bovine serum (FBS) were obtained from Gibco. Cells were maintained on 
a
GMR-GAL4

UAS127Q
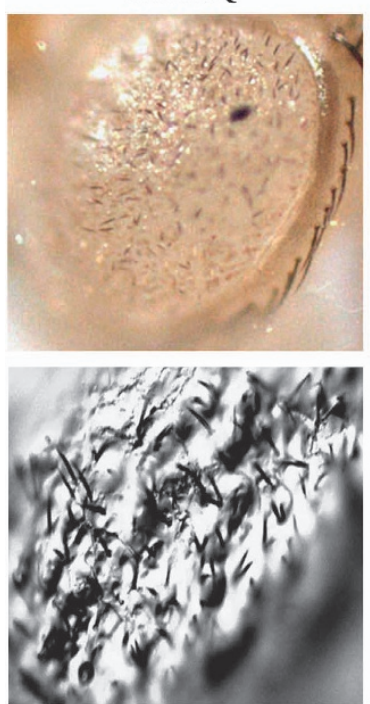

GMR-GAL4

UAS127Q;

UASdCUL1-RNAi
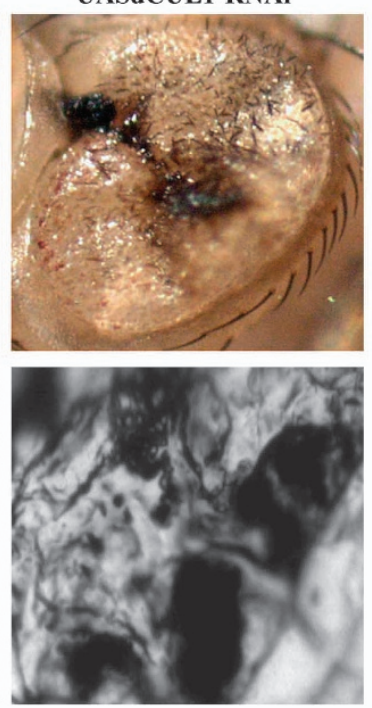

DAPI

b
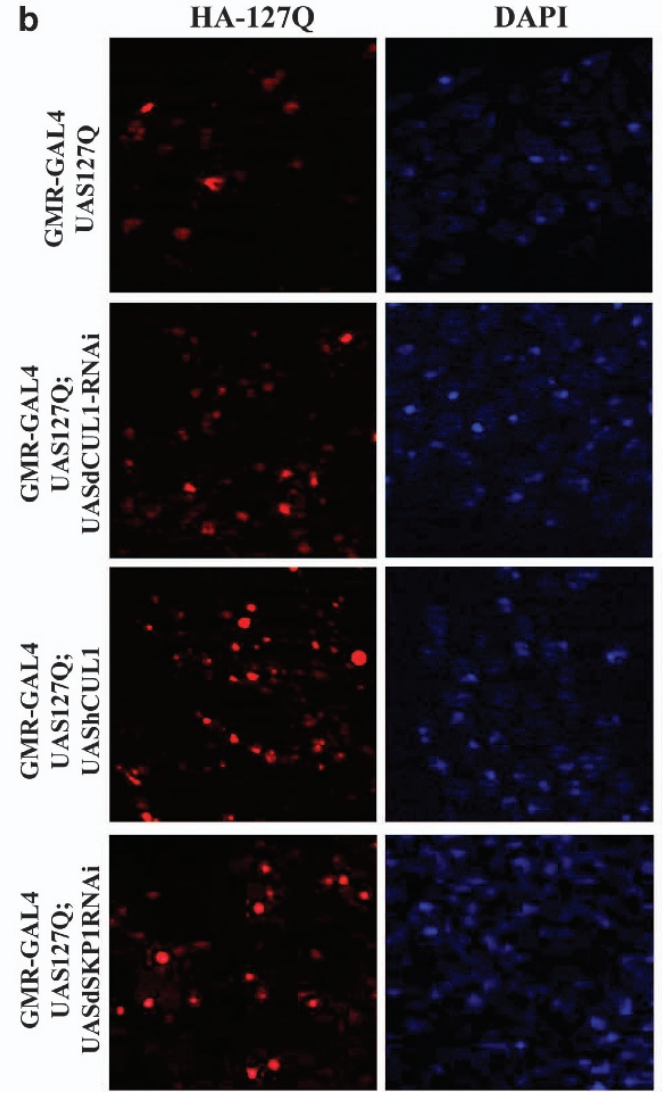

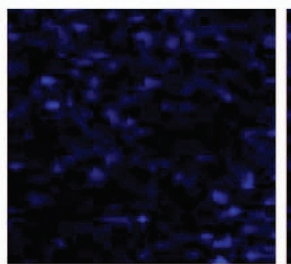

GMR-GAL4

UAS127Q;

UASdSKP1-RNAi
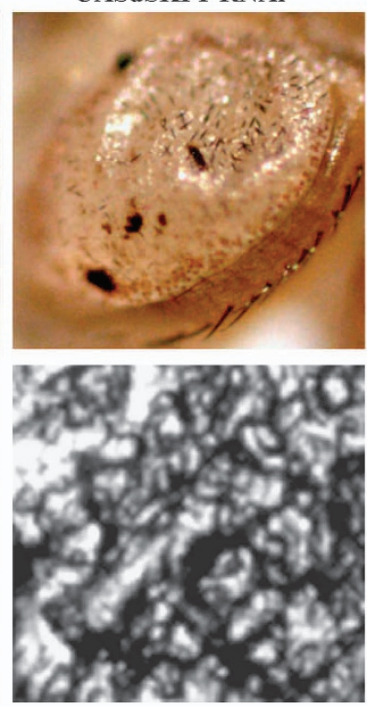

MERGED
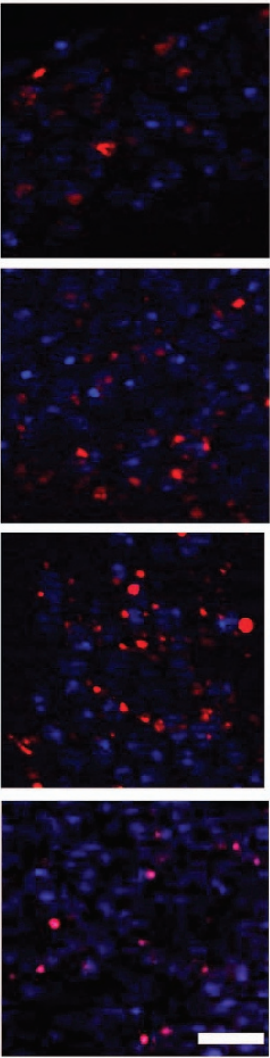

Figure 8 Effect of modulating dCul1 and dSkp1 levels on polyQ expressing transgenic flies. (a) External morphology (top panel) and nail polish imprints (bottom panel) of the eye surface of Drosophila with dCul1 or dSkp1 RNAi in the presence of 127 polyQ repeats compared with GMR-GAL4.UAS127Q controls. Downregulating both dCul1 and dSkp1 enhances polyQ toxicity as seen in the worsening of the degenerate eyes and appearance of black spots. (b) Immunostaining of HA-tagged 127 polyQ aggregates (using HA antibody) in the developing eye imaginal disc of third instar larvae. Downregulation of dCul1 or dSkp1 and overexpression of hCul1 results in an increase in aggregate load. Scale bar: $10 \mu \mathrm{m}$

DMEM supplemented with heat-inactivated $10 \%$ FBS and antibiotics $(0.4 \mathrm{mg} / \mathrm{ml}$ Zeocin and $0.4 \mathrm{mg} / \mathrm{ml} \mathrm{G} 418)$. They were transiently transfected with pcDNA3-mycCUL1 or pcDNA3.1 (empty vector) using Lipofectamine 2000 (Invitrogen,
Carlsbad, CA, USA) reagent according to the manufacturer's instructions. Production of GFP-tagged tNhtt-150Q aggregates was induced for the indicated time using $1 \mu \mathrm{M}$ ponasterone A (Invitrogen). 
Immunostaining and quantification of aggregates. Cells were fixed in $4 \%$ PFA for $30 \mathrm{~min}$ at room temperature and then processed for immunofluorescence as described previously. ${ }^{43}$ Endogenous Cul1 was stained using rabbit anti-Cul1 (1:1000, Abcam) and transfected Cul1 using mouse anti-cmyc (1:1000, Santa Cruz Biotechnology, Santa Cruz, CA, USA). Alexafluor 594 conjugated anti-rabbit or anti-mouse (1:500, Invitrogen) was used as secondary antibody. To count aggregates, cells were grown in two-well chamber slides, transfected with myc-hCUL1 and aggregates were induced with ponasterone A for $12 \mathrm{~h}$. Random images were taken across regions of each chamber such that at least 75 transfected and control cells each could be counted per chamber. Four independent chambers were used to compare the percentage of transfected cells containing aggregates to control.

Fly stocks. Fly stocks were maintained at $25 \pm 1^{\circ} \mathrm{C}$ in $\mathrm{BOD}$ incubators on standard sugar yeast food containing nipagin and propionic acid to prevent bacterial and fungal contaminations. The RNAi stocks were obtained from VDRC (stock numbers - 42445, 108558, 33406 for dCul1 and 46605, 46607 for dSkp1). Flies overexpressing hCul1 $\left(w^{-} ;+;\right.$UAShCUL1/TM6B) were generated in this study. Other stocks used in this study were: OregonR ${ }^{+}, w^{-}$; GMR-GAL4;+, $w^{-} ;$UASHTT-EX1-PQ93/CyO;+, $w^{-} ;$UASMJD-PQ78(s);+ and $w^{-} ;$GMRGAL4 UAS127Q/CyO; +.

Generating flies overexpressing hCul1. pcDNA3-myc-CUL1 (plasmid number 19896, Addgene, Cambridge, MA, USA) contains the coding sequence for all 752 amino acids of human Cul1 (approximately $87 \mathrm{kDa}$ protein) along with myc tag at the N-terminal end. The Drosophila transformation vector, pUASTattB, was obtained from FlyC31. The hCul1 insert (without the myc tag) was digested from pcDNA3-myc3-CUL1 using BamHI (Roche, Penzberg, Germany). pUASTattB was linearised using Bgll (Roche) to provide BamHI compatible ends. The two fragments were gel-purified using a Qiagen gel extraction kit and were ligated using T4 DNA ligase (Invitrogen). After transformation, the orientation of the insert was checked by restriction digestion and the correct clone was sent to Transgenic Fly Facility (TFF, C-CAMP, Bangalore) for generating transgenic lines.

Examination of fly eye structure. The external gross morphology of the eye was imaged on a Leica DM RXA2 microscope using a $\times 5$ objective. The structure of ommatidia was analysed using nail polish imprints. ${ }^{33}$ Briefly, anesthetised flies were decapitated and their heads were dipped in a drop of nail polish and allowed to dry. As the nail polish hardens, it forms an imprint of the eye and this imprint was peeled off and imaged using bright-field microscopy.

Whole-organ immunostaining and microscopy. Eye imaginal discs of third instar larvae of the desired genotypes were dissected in PBS and fixed in 4\% PFA for $45 \mathrm{~min}$. They were then processed for immunostaining using standard protocols. Mouse anti-HA (Roche) was used as the primary antibody at a dilution of 1:1000. Anti-mouse secondary antibody, conjugated to AlexaFluor 594 (Invitrogen), was used at a dilution of 1:1000. The tissue was mounted in DAPImounting medium (Vector Laboratories). Z-series images were taken on an ApoTome (Carl Zeiss, Oberkochen, Germany) microscope using a $\times 63$ oil objective (1.4 NA) and processed using ImageJ (NIH, Bethesda, MD, USA) and Adobe Photoshop (Adobe Systems, San Jose, CA, USA). When comparing aggregate load between genotypes, all microscope settings were kept constant.

Immunoblotting. Protein was extracted from Drosophila heads in lysis buffer

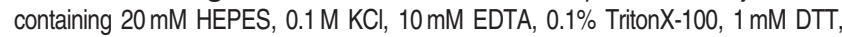
$5 \%$ Glycerol and complete protease inhibitor cocktail (Roche). HD-150Q cells were pelletted and lysed in NP-40 lysis buffer (50 mM Tris, pH 8.0, $150 \mathrm{mM} \mathrm{NaCl}, 1 \% \mathrm{NP}$ 40 and complete protease inhibitor cocktail) and briefly sonicated. Mice brain parts were carefully dissected and homogenised in NP-40 lysis buffer. All lysed protein samples were centrifuged at $15000 \times g$ for $10 \mathrm{~min}$ and supernatant was used for immunoblotting. Primary antibodies used were rabbit anti-Cul1 (1:1000, Invitrogen), rabbit anti-Skp1 (1:500, Cell Signalling), mouse anti-c-myc (1:1000, Santa Cruz Biotechnology), rabbit anti- $\beta$-catenin (1:1000, Santa Cruz Biotechnology), mouse $\beta$ actin (1:5000, Abcam) and mouse anti-GAPDH (1:5000, Santa Cruz Biotechnology). HRP-conjugated secondary antibodies (Vector Laboratories) were used and blots were developed using Immobilon Western Chemiluminescent HRP Substrate (Millipore, Billerica, MA, USA). ImageJ was used to quantify the signal from immunoblots. The value obtained for the protein of interest (Cul1 or Skp1) was normalised to the loading control (GAPDH or $\beta$-actin). The fold change represented has been calculated in comparison with control for each experiment.

RT-PCR. Total RNA was extracted from heads of flies by using Trizol reagent (Sigma) and following manufacturer's instructions. Total RNA was quantified and $75 \mathrm{ng}$ was used in each RT-PCR reaction. dcul1 RNA was amplified using the following primers - Forward primer 5'-AGCGCCGAATTAACATCAAC-3'; Reverse primer $5^{\prime}$-ACAGCCCCTGCAGAAGTCTA-3' ${ }^{44}$ RP49 was used as a loading control and amplified using Forward primer 5'-AGCGCACCAAGCACTTCA TCCGCCA-3'; Reverse primer $5^{\prime}$-GCGCACGTTGTGCACCAGGAACTTC- $3^{\prime}{ }^{\prime 4}$ RT-PCR was done using one-step Superscript III Reverse Transcriptase kit (Invitrogen). PCR product was run on an ethidium bromide agarose gel, and band intensity was quantified using ImageJ.

Data analysis. All images were arranged using Adobe Photoshop, graphs were made in SigmaPlot and tables in Microsoft Word. All statistical data were obtained by conducting either one-way ANOVAs followed by post-hoc test or twotailed Student's $t$-tests as indicated in figure legends. Values are expressed as mean \pm S.E.M. $P<0.05$ was considered statistically significant.

\section{Conflict of Interest}

The authors declare no conflict of interest.

Acknowledgements. This work was funded by a core grant from the Department of Biotechnology, Government of India to National Brain Research Centre. We thank Mr Ankit Sharma for technical assistance.

\section{Author Contributions}

$\mathrm{SB}$ and $\mathrm{NRJ}$ designed the experiments; $\mathrm{SB}, \mathrm{AD}$ and $\mathrm{MM}$ performed the experiments; SCL contributed reagents/materials; and SB, SCL and NRJ wrote the manuscript.

1. MacDonald ME, Ambrose CM, Duyao MP, Myers RH, Lin C, Srinidhi L et al. A novel gene containing a trinucleotide repeat that is expanded and unstable on Huntington's disease chromosomes. The Huntington's Disease Collaborative Research Group. Cell 1993; 72 : 971-983.

2. Rubinsztein DC, Leggo J, Coles R, Almqvist E, Biancalana V, Cassiman JJ et al. Phenotypic characterization of individuals with 30-40 CAG repeats in the Huntington disease (HD) gene reveals $\mathrm{HD}$ cases with 36 repeats and apparently normal elderly individuals with 36-39 repeats. Am J Hum Genet 1996; 59: 16-22.

3. Kawaguchi $\mathrm{Y}$, Okamoto T, Taniwaki M, Aizawa M, Inoue M, Katayama S et al. CAG expansions in a novel gene for Machado-Joseph disease at chromosome 14q32.1. Nat Genet 1994; 8: 221-228.

4. Soto $\mathrm{C}$. Unfolding the role of protein misfolding in neurodegenerative diseases. Nat Rev Neurosci 2003; 4: 49-60.

5. Ross CA, Tabrizi SJ. Huntington's disease: from molecular pathogenesis to clinical treatment. Lancet Neurol 2011; 10: 83-98.

6. Jackson GR, Salecker I, Dong X, Yao X, Arnheim N, Faber PW et al. Polyglutamineexpanded human huntingtin transgenes induce degeneration of Drosophila photoreceptor neurons. Neuron 1998; 21: 633-642.

7. Mallik M, Lakhotia SC. Modifiers and mechanisms of multi-system polyglutamine neurodegenerative disorders: lessons from fly models. J Genet 2010; 89: 497-526.

8. Wang GH, Mitsui K, Kotliarova S, Yamashita A, Nagao Y, Tokuhiro S et al. Caspase activation during apoptotic cell death induced by expanded polyglutamine in N2a cells. Neuroreport 1999; 10: 2435-2438.

9. Mangiarini L, Sathasivam K, Seller M, Cozens B, Harper A, Hetherington C et al. Exon 1 of the $\mathrm{HD}$ gene with an expanded $C A G$ repeat is sufficient to cause a progressive neurological phenotype in transgenic mice. Cell 1996; 87: 493-506.

10. Lehman NL. The ubiquitin proteasome system in neuropathology. Acta Neuropathol 2009; 118: 329-347.

11. Jana NR, Zemskov EA, Wang G, Nukina N. Altered proteasomal function due to the expression of polyglutamine-expanded truncated $\mathrm{N}$-terminal huntingtin induces apoptosis by caspase activation through mitochondrial cytochrome c release. Hum Mol Genet 2001; 10: $1049-1059$.

12. Kitada T, Asakawa S, Hattori N, Matsumine H, Yamamura Y, Minoshima S et al. Mutations in the parkin gene cause autosomal recessive juvenile parkinsonism. Nature 1998; 392 : 605-608.

13. Cummings CJ, Reinstein E, Sun Y, Antalffy B, Jiang Y, Ciechanover A et al. Mutation of the E6-AP ubiquitin ligase reduces nuclear inclusion frequency while accelerating polyglutamine-induced pathology in SCA1 mice. Neuron 1999; 24: 879-892. 
14. Maheshwari M, Samanta A, Godavarthi SK, Mukherjee R, Jana NR. Dysfunction of the ubiquitin ligase ube3a may be associated with synaptic pathophysiology in a mouse model of huntington disease. J Biol Chem 2012; 287: 29949-29957.

15. Mishra A, Dikshit P, Purkayastha S, Sharma J, Nukina N, Jana NR. E6-AP promotes misfolded polyglutamine proteins for proteasomal degradation and suppresses polyglutamine protein aggregation and toxicity. J Biol Chem 2008; 283: 7648-7656.

16. Jana NR, Dikshit P, Goswami A, Kotliarova S, Murata S, Tanaka K et al. Co-chaperone CHIP associates with expanded polyglutamine protein and promotes their degradation by proteasomes. J Biol Chem 2005; 280: 11635-11640.

17. Tsai YC, Fishman PS, Thakor NV, Oyler GA. Parkin facilitates the elimination of expanded polyglutamine proteins and leads to preservation of proteasome function. $J$ Biol Chem 2003; 278: 22044-22055.

18. Skowyra D, Craig KL, Tyers M, Elledge SJ, Harper JW. F-box proteins are receptors that recruit phosphorylated substrates to the SCF ubiquitin-ligase complex. Cell 1997; 91: 209-219.

19. Latres E, Chiaur DS, Pagano M. The human F box protein beta-Trcp associates with the Cul1/Skp1 complex and regulates the stability of beta-catenin. Oncogene 1999; 18 849-854.

20. Hori T, Osaka F, Chiba T, Miyamoto C, Okabayashi K, Shimbara N et al. Covalent modification of all members of human cullin family proteins by NEDD8. Oncogene 1999; 18: 6829-6834.

21. Skaar JR, Pagano M. Control of cell growth by the SCF and APC/C ubiquitin ligases. Curr Opin Cell Biol 2009; 21: 816-824.

22. Uro-Coste E, Fonta C, Hatey F, Perret E, Delisle MB, Caput D et al. Expression of SKP1 mRNA and protein in rat brain during postnatal development. Neuroreport 1997; 8: 1675-1678.

23. Michel JJ, Xiong Y. Human CUL-1, but not other cullin family members, selectively interacts with SKP1 to form a complex with SKP2 and cyclin A. Cell Growth Differ 1998; 9: 435-449.

24. Staropoli JF, Abeliovich A. The ubiquitin-proteasome pathway is necessary for maintenance of the postmitotic status of neurons. J Mol Neurosci 2005; 27: 175-183.

25. Fishman-Jacob T, Reznichenko L, Youdim MB, Mandel SA. A sporadic Parkinson disease model via silencing of the ubiquitin-proteasome/E3 ligase component SKP1A. J Biol Chem 2009; 284: 32835-32845.

26. Fishman-Jacob T, Youdim MB, Mandel SA. Silencing/overexpressing selected genes as a model of sporadic Parkinson's disease. Neurodegener Dis 2010; 7: 108-111.

27. Mandel SA, Fishman-Jacob T, Youdim MB. Targeting Skp1, an ubiquitin E3 ligase component found decreased in sporadic Parkinson's disease. Neurodegener Dis 2011; 10: 220-223.

28. Mandel SA, Fishman-Jacob T, Youdim MB. Genetic reduction of the E3 ubiquitin ligase element, SKP1A and environmental manipulation to emulate cardinal features of Parkinson's disease. Parkinsonism Relat Disord 2012; 18(Suppl 1): S177-S179.

29. Doumanis J, Wada K, Kino Y, Moore AW, Nukina N. RNAi screening in Drosophila cells identifies new modifiers of mutant huntingtin aggregation. PLoS One 2009; 4: e7275.

30. Zhang S, Binari R, Zhou R, Perrimon N. A genomewide RNA interference screen for modifiers of aggregates formation by mutant Huntingtin in Drosophila. Genetics 2010; 184 $1165-1179$

31. Heriche JK, Ang D, Bier E, O'Farrell PH. Involvement of an SCFSImb complex in timely elimination of E2F upon initiation of DNA replication in Drosophila. BMC Genet 2003; 4: 9.
32. Voigt J, Chen JA, Gilchrist M, Amaya E, Papalopulu N. Expression cloning screening of a unique and full-length set of cDNA clones is an efficient method for identifying genes involved in Xenopus neurogenesis. Mech Dev 2005; 122: 289-306.

33. Mallik M, Lakhotia SC. The developmentally active and stress-inducible noncoding hsromega gene is a novel regulator of apoptosis in Drosophila. Genetics 2009; 183: 831-852.

34. Warrick JM, Paulson HL, Gray-Board GL, Bui QT, Fischbeck KH, Pittman RN et al. Expanded polyglutamine protein forms nuclear inclusions and causes neural degeneration in Drosophila. Cell 1998; 93: 939-949.

35. Ou CY, Lin YF, Chen YJ, Chien CT. Distinct protein degradation mechanisms mediated by Cul1 and Cul3 controlling Ci stability in Drosophila eye development. Genes Dev 2002; 16 : 2403-2414.

36. Riley BE, Orr HT. Polyglutamine neurodegenerative diseases and regulation of transcription: assembling the puzzle. Genes Dev 2006; 20: 2183-2192.

37. Yen HC, Elledge SJ. Identification of SCF ubiquitin ligase substrates by global protein stability profiling. Science 2008; 322: 923-929.

38. Copani A, Uberti D, Sortino MA, Bruno V, Nicoletti F, Memo M. Activation of cell-cycleassociated proteins in neuronal death: a mandatory or dispensable path? Trends Neurosci 2001; 24: 25-31.

39. Godin JD, Poizat G, Hickey MA, Maschat F, Humbert S. Mutant huntingtin-impaired degradation of beta-catenin causes neurotoxicity in Huntington's disease. EMBO J 2010; 29: 2433-2445.

40. Mori F, Nishie M, Piao YS, Kito K, Kamitani T, Takahashi H et al. Accumulation of NEDD8 in neuronal and glial inclusions of neurodegenerative disorders. Neuropathol Appl Neurobiol 2005; 31: 53-61.

41. Dil Kuazi A, Kito K, Abe Y, Shin RW, Kamitani T, Ueda N. NEDD8 protein is involved in ubiquitinated inclusion bodies. J Pathol 2003; 199: 259-266.

42. Mehta R, Steinkraus KA, Sutphin GL, Ramos FJ, Shamieh LS, Huh A et al. Proteasomal regulation of the hypoxic response modulates aging in C. elegans. Science 2009; 324: 1196-1198.

43. Mishra A, Godavarthi SK, Maheshwari M, Goswami A, Jana NR. The ubiquitin ligase E6-AP is induced and recruited to aggresomes in response to proteasome inhibition and may be involved in the ubiquitination of Hsp70-bound misfolded proteins. $\mathrm{J}$ Biol Chem 2009; 284: 10537-10545.

44. Zhou D, Xue J, Chen J, Morcillo P, Lambert JD, White KP et al. Experimental selection for Drosophila survival in extremely low O(2) environment. PLOS One 2007; 2: e490.

45. Fujikake N, Nagai Y, Popiel HA, Okamoto Y, Yamaguchi M, Toda T. Heat shock transcription factor 1-activating compounds suppress polyglutamine-induced neurodegeneration through induction of multiple molecular chaperones. J Biol Chem 2008; 283 26188-26197.

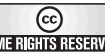

Cell Death and Disease is an open-access journal published by Nature Publishing Group. This work is licensed under the Creative Commons Attribution-NonCommercial-No Derivative Works 3.0 Unported License. To view a copy of this license, visit http://creativecommons.org/licenses/by-nc-nd/3.0/

Supplementary Information accompanies the paper on Cell Death and Disease website (http://www.nature.com/cddis) 